\section{Describing the burden of the COVID-19 pandemic in people with psoriasis: findings from a global cross-sectional study}

\section{Editor,}

Indirect excess morbidity in the COVID-19 pandemic may arise from public health risk-mitigation efforts such as stay-at-home orders and re-purposing of healthcare services. ${ }^{1}$ Increased mental health disorders and shortfalls in the care of long-term conditions are described. ${ }^{2,3}$ We used global self-reported crosssectional data to characterise the factors associated with worsening psoriasis in the pandemic, focussing on the impact of anxiety and depression.

Data from a cross-sectional survey (PsoProtect $M e^{4}$ ) were extracted on 15th January 2021. After excluding participants self-reporting COVID-19, the association between mental health and worsening psoriasis was assessed using a fully adjusted logistic regression model including covariates selected a priori as potentially influential on psoriasis severity and anxiety/depression. Participants scoring $\geq 3$ in GAD-2 or PHQ- 2 defined a positive mental health screen. ${ }^{5}$

4043 people with psoriasis from 86 countries were included (Table 1). $1728(42.7 \%)$ reported worsening psoriasis in the pandemic. A total of $3575(88.4 \%)$ returned information on their mental health, with a greater proportion of those reporting worsening psoriasis having a positive mental health screen (814/ $1621,50.2 \%)$ compared to those without worsening psoriasis (562/1954, 28.8\%). A greater proportion of females reported worsening psoriasis $(1322 / 2684,49.3 \%)$ compared with males (406/1354, 30.0\%).

A fully adjusted regression model for worsening psoriasis estimated an odds ratio (OR) 2.01 (95\% CI, 1.72-2.34) for those with a positive screen for anxiety or depression compared to

Table 1 Patient demographics and clinical characteristics stratified by disease state

\begin{tabular}{|c|c|c|c|c|c|}
\hline & $\begin{array}{l}\text { Total } \\
N=4,043\end{array}$ & Missing data & $\begin{array}{l}\text { Non-worsening disease } \\
N=2,315\end{array}$ & $\begin{array}{l}\text { Worsening disease } \\
N=1,728\end{array}$ & $P$-value \\
\hline Shielded & $2,224(55.1 \%)$ & $9(0.2 \%)$ & $1,240(53.8 \%)$ & $984(56.9 \%)$ & 0.045 \\
\hline Advised to shield & $742(33.6 \%)$ & $1,833(45.3 \%)$ & $465(37.5 \%)$ & $277(28.6 \%)$ & $<0.001$ \\
\hline Female gender & $2,684(66.5 \%)$ & $5(0.1 \%)$ & $1,362(59.0 \%)$ & $1,322(76.5 \%)$ & $<0.001$ \\
\hline Age, mean (SD) & $47.2(15.1)$ & $31(0.8 \%)$ & $49.5(15.3)$ & $44.2(14.3)$ & $<0.001$ \\
\hline White European ethnicity & $3,016(74.6 \%)$ & 0 & $1,707(73.7 \%)$ & $1,309(75.8 \%)$ & 0.15 \\
\hline BMI, mean (SD) & $27.6(6.0)$ & $369(9.1 \%)$ & $27.4(5.8)$ & $28.0(6.3)$ & 0.003 \\
\hline Alcohol $>14$ units a week & $495(13.8 \%)$ & $455(11.3 \%)$ & $295(15.0 \%)$ & $200(12.3 \%)$ & 0.018 \\
\hline Current smoker & $559(15.8 \%)$ & $498(12.3 \%)$ & $291(15.0 \%)$ & $268(16.7 \%)$ & 0.18 \\
\hline Full time employed & $1,929(47.7 \%)$ & 0 & $1,072(46.3 \%)$ & 857 (49.6\%) & 0.038 \\
\hline Household number, mean (SD) & $2.8(1.8)$ & $26(0.6 \%)$ & $2.8(1.7)$ & $2.9(1.9)$ & 0.003 \\
\hline Key worker & $1,131(28.1 \%)$ & $22(0.5 \%)$ & $595(25.9 \%)$ & $536(31.1 \%)$ & $<0.001$ \\
\hline Psoriasis severity prior to COVID-19 pandemic & & $284(7 \%)$ & & & $<0.001$ \\
\hline Clear & $451(12.0 \%)$ & & $299(14.6 \%)$ & $152(8.9 \%)$ & \\
\hline Nearly clear & $767(20.4 \%)$ & & $463(22.7 \%)$ & $304(17.7 \%)$ & \\
\hline Mild & $989(26.3 \%)$ & & $477(23.3 \%)$ & $512(29.9 \%)$ & \\
\hline Moderate & $892(23.7 \%)$ & & $442(21.6 \%)$ & $450(26.2 \%)$ & \\
\hline Moderate-severe & $480(12.8 \%)$ & & $273(13.4 \%)$ & $207(12.1 \%)$ & \\
\hline Severe & $180(4.8 \%)$ & & $90(4.4 \%)$ & $90(5.2 \%)$ & \\
\hline Systemic therapy & & $522(12.9 \%)$ & & & $<0.001$ \\
\hline No systemic therapy & $1,980(56.2 \%)$ & & $938(49.4 \%)$ & $1,042(64.2 \%)$ & \\
\hline Standard systemic therapy & $560(15.9 \%)$ & & $309(16.3 \%)$ & $251(15.5 \%)$ & \\
\hline Targeted therapy & $981(27.9 \%)$ & & $652(34.3 \%)$ & $329(20.3 \%)$ & \\
\hline Non adherent to systemic therapy & $284(18.4 \%)$ & $2507(62 \%)$ & $114(11.9 \%)$ & $170(29.6 \%)$ & $<0.001$ \\
\hline 1 or more comorbidity & $1,606(39.7 \%)$ & 0 & $908(39.2 \%)$ & $698(40.4 \%)$ & 0.45 \\
\hline Anxiety & $1,069(30.1 \%)$ & $489(12.1 \%)$ & $408(21.0 \%)$ & $661(41.0 \%)$ & $<0.001$ \\
\hline Depression & $977(27.5 \%)$ & $494(12.2 \%)$ & $392(20.1 \%)$ & $585(36.5 \%)$ & $<0.001$ \\
\hline Anxiety or depression & $1,376(38.5 \%)$ & $468(11.6 \%)$ & $562(28.8 \%)$ & $814(50.2 \%)$ & $<0.001$ \\
\hline
\end{tabular}

Targeted therapy was defined as anyone taking TNF inhibitors (adalimumab, certolizumab, etanercept, infliximab), IL-17 inhibitors (ixekizumab, secukinumab, brodalumab), IL-23 inhibitors (guselkumab, risankizumab, ustekinumab; apremilast). Standard systemic therapy was defined as anyone taking acitretin, ciclosporin, or methotrexate and not taking a targeted therapy.

BMI, body mass index; SD, standard deviation. 


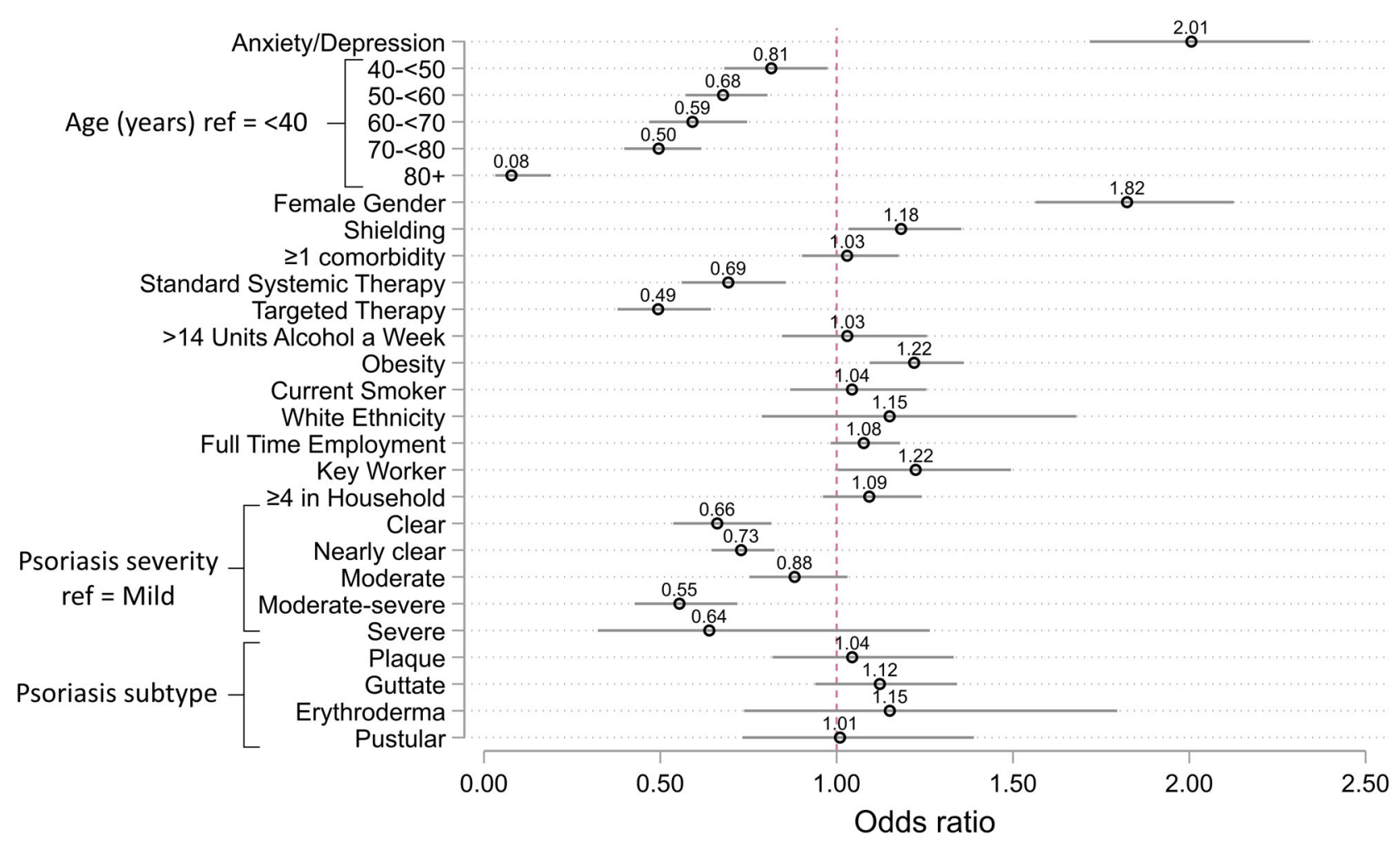

Figure 1 Fully adjusted model for associations with worsening psoriasis. Odds ratios for associations with worsening psoriasis. Anxiety/ depression is defined as those who screened positive for either anxiety or depression. Obesity is defined as a BMI $>30$.

those without a positive screen (Fig. 1). Associations were also observed for female gender (OR, 1.82, 95\% CI, 1.56-2.13); obesity (OR, 1.22, 95\% CI, 1.09-1.36) and shielding (OR, 1.18, 95\% CI, 1.03-1.35).

There were inverse associations with systemic therapy use [standard systemic OR 0.69 [95\% CI, 0.56-0.86] and targeted therapy OR 0.49 (95\% CI, 0.38-0.64)]. Of 1541 (38.1\%) participants receiving standard systemic or targeted therapies for psoriasis, 284 (18.4\%) reported non-adherence during the pandemic (Table 1). The commonest reason was concern regarding complications related to COVID-19 $(n=217)$. Non-adherence was associated with worsening psoriasis (OR, 2.90, 95\% CI, 2.313.63). A positive mental health screen was more common in those reporting non-adherence compared to those who were adherent ( $42.8 \%$ vs. $32.4 \%$ ).

These data indicate a burden due to the COVID-19 pandemic in people with psoriasis; worsening psoriasis is common and is associated with poor mental health. We find that in the subset on systemic therapy, non-adherence is associated with worsening disease and is driven by concerns about immunosuppressant-related risks of COVID-19. This is an important observation since current guidelines (informed by reassuring data on drug-related risks of severe COVID-19 $9^{6}$ ) recommend continuing immunosuppression in people without COVID-19 to maintain disease control. ${ }^{7}$
Our findings parallel data from the general population indicating an increased mental health burden during the pandemic, particularly in women. ${ }^{8}$ People with psoriasis - especially those with severe psoriasis, and women - have a high prevalence of anxiety and depression and may thus be particularly vulnerable to the adverse impact of the pandemic on mental health. ${ }^{9}$ Whilst men are known to be at greater risk of severe outcomes from COVID-19, our data suggest that women may be more susceptible to indirect excess morbidity - poor mental health and worsening skin disease - than men.

The generalisability of results is limited given the self-selecting bias of our study population towards UK white women. Individuals non-adherent to treatment, with low computer literacy or less anxiety, may be disinclined to participate, which may introduce ascertainment bias.

Our data underscore the importance of holistic models of care and indicate a need to provide access to psychological support. In those with worsening psoriasis, possible non-adherence should be explored. Evidence-based communication around medication-related COVID-19 risks and behavioural approaches for supporting adherence may help address fears, anxieties and confusion. ${ }^{10}$ Attention given now to address this may mitigate a long-lasting detrimental impact of the pandemic on health outcomes in people with psoriasis. 


\section{Acknowledgements}

We are grateful to all the patients who have contributed to PsoProtect $M e$, the professional and patient organisations who supported or promoted PsoProtectMe and for the input of Prof Lars Iversen, Prof Nick Reynolds, Prof Joel Gelfand, Ms Christine Janus and Ms Melissa Sweeney through their vital contributions. We would also like to acknowledge the following individuals for help with translating the PsoProtectMe survey; Dr Haleema Alfailakawi, Dr Wisam Alwan, Dr Rosa Andres Ejarque, Dr Ines Barbosa, Ms Carmen Bugarin Diz, Ms Katarzyna Grys, Dr Mahira Hamdy El Sayed, Mr Tran Hong Truong, Mr Masanori Okuse, Ms Dagmara Samselska, Ms Isabella Tosi, Ms Ya-Hsin Wang, and the Engine Group UK for their generous creative input and website expertise.

\section{Conflict of interest}

Nothing to disclose: Dr Yates, Dr Dand, Prof. Langan, Dr. Norton, Dr. Tsakok, Dr. Yiu, Dr De La Cruz, Dr. Contreras, Ms. Vesty, Ms. Vincent, Mr. Bola Coker, Ms. Meynell, Dr. Lambert, Prof. Brown, Prof. Naldi. Prof. Barker reports grants and personal fees from Abbvie, grants and personal fees from Novartis, grants and personal fees from Lilly, grants and personal fees from J\&J, from null, during the conduct of the study. Prof. Griffiths reports grants and personal fees from AbbVie, grants from Amgen, grants from BMS, grants and personal fees from Janssen, grants from LEO, grants and personal fees from Novartis, grants from Pfizer, grants from Almirall, grants and personal fees from Lilly, grants and personal fees from UCB Pharma, outside the submitted work. Prof. Jullien reports personal fees and non-financial support from Abbvie, personal fees and nonfinancial support from Novartis, personal fees and non-financial support from Janssen-Cilag, personal fees and non-financial support from Lilly, personal fees and non-financial support from Leo-Pharma, personal fees and non-financial support from MEDAC, personal fees and non-financial support from Celgene, personal fees from Amgen, outside the submitted work. Dr. Capon reports consultancy fees from AnaptysBio, grants from Boheringer-Ingelheim, outside the submitted work. Prof. Bachelez reports personal fees from Abbvie, personal fees from Janssen, personal fees from LEO Pharma, personal fees from Novartis, personal fees from UCB, personal fees from Almirall, personal fees from Biocad, personal fees from Boehringer-Ingelheim, personal fees from Kyowa Kirin, personal fees from Pfizer, outside the submitted work. Prof. Gisondi reports personal fees from Abbvie, Amgen, Eli Lilly, Janssen, Novartis, Pierre Fabre, Sandoz, UCB, outside the submitted work. Dr. Galloway reports personal fees from Abbvie, personal fees from Sanofi, personal fees from Novartis, personal fees from Pfizer, grants from Eli Lilly, personal fees from Janssen, personal fees from UCB, outside the submitted work. Prof. Weinmann has presented talks for Abbvie, Abbott, Bayer, Chiesi, Boehringer
Ingelheim, Roche and Merck. Dr. Mason reports personal fees from LEO Pharma and Novartis, outside the submitted work. Ms. Moorhead reports personal fees from Abbvie, personal fees from Celgene, personal fees from Janssen, personal fees from LEO Pharma, personal fees from Novartis, personal fees from UCB, outside the submitted work. Dr. Puig reports grants and personal fees from AbbVie, grants and personal fees from Almirall, grants and personal fees from Amgen, grants and personal fees from Boehringer Ingelheim, personal fees from Bristol Myers Squibb, personal fees from Fresenius-Kabi, grants and personal fees from Janssen, grants and personal fees from Lilly, personal fees from Mylan, grants and personal fees from Novartis, personal fees from Pfizer, personal fees from Sandoz, personal fees from Sanofi, personal fees from Samsung-Bioepis, grants and personal fees from UCB, outside the submitted work. Dr. Mahil reports departmental income from Abbvie, Celgene, Eli Lilly, Janssen-Cilag, Novartis, Sanofi, UCB, outside the submitted work. Dr. Di Meglio reports grants and personal fees from UCB, personal fees from Novartis, personal fees from Janssen, outside the submitted work. Prof. Warren reports grants and personal fees from Abbvie, grants and personal fees from Celgene, grants and personal fees from Eli Lilly, grants and personal fees from Novartis, personal fees from Sanofi, grants and personal fees from UCB|, grants and personal fees from Almirall, grants and personal fees from Amgen, grants and personal fees from Janssen, grants and personal fees from Leo, grants and personal fees from Pfizer, personal fees from Arena, personal fees from Avillion, personal fees from Bristol Myers Squibb, personal fees from Boehringer Ingelheim, outside the submitted work. Prof. Smith reports grants from Abbvie, Sanofi, Novartis, and Pfizer and through consortia with multiple academic partners (psort.org.uk, BIOMAP-IMI.eu), outside the submitted work. Dr. Torres reports grants and personal fees from AbbVie, Almirall, Amgen, Arena Pharmaceuticals, Biogen, Biocad, Boehringer Ingelheim, Bristol-Myers Squibb, Celgene, Eli Lilly, Janssen, LEO Pharma, MSD, Novartis, Pfizer, Samsung-Bioepis, Sandoz, during the conduct of the study. Dr. Waweru is on the Board of the International Federation of Psoriasis Associations who have received grants from Abbvie, Almirall, Amgen, Bristol Meyers Squibb, Boehringer Ingelheim, Celgene, Janssen, Leo Pharma, Eli Lilly, Novartis, Sun Pharma, Pfizer, and UCB, outside the submitted work. Mr. Urmston reports grants from Almirall, grants from Abbvie, grants from Amgen, grants from Celgene, grants from Dermal Laboratories, grants from Eli Lilly, grants from Janssen, grants from LEO Pharma, grants from T and R Derma, grants from UCB, outside the submitted work. Ms. McAteer reports grants from Abbvie, grants from Almirall, grants from Amgen, grants from Celgene, grants from Dermal Laboratories, grants from Eli Lilly, grants from Janssen, grants from LEO Pharma, grants from UCB, grants from $\mathrm{T}$ and R Derma, outside the submitted work. 


\section{Funding sources}

We acknowledge financial support from the Department of Health via the National Institute for Health Research (NIHR) Biomedical Research Centre based at Guy's and St Thomas' NHS Foundation Trust and King's College London, the NIHR Manchester Biomedical Research Centre and the Psoriasis Association. The views expressed are those of the author (s) and not necessarily those of the NHS, the NIHR, or the Department of Health and Social Care. SKM is funded by a Medical Research Council (MRC) Clinical Academic Research Partnership award (MR/T02383X/1). ND is funded by Health Data Research UK (MR/S003126/1), which is funded by the UK MRC, Engineering and Physical Sciences Research Council; Economic and Social Research Council; Department of Health \& Social Care (England); Chief Scientist Office of the Scottish Government Health and Social Care Directorates; Health and Social Care Research and Development Division (Welsh Government); Public Health Agency (Northern Ireland); British Heart Foundation; and Wellcome Trust. ZZNY is funded by an NIHR Academic Clinical Lectureship through the University of Manchester. CEMG is an NIHR Emeritus Senior Investigator and is funded in part by the MRC (MR/ 101 1808/1). CEMG and RBW are in part supported by the NIHR Manchester Biomedical Research Centre. SML is supported by a Wellcome senior research fellowship in clinical science $(205039 / \mathrm{Z} / 16 / \mathrm{Z})$; this research was funded in whole or in part by the Wellcome Trust [205039/Z/16/Z]. For the purpose of Open Access, the author has applied a CC BY public copyright licence to any Author Accepted Manuscript (AAM) version arising from this submission. SML is also supported by Health Data Research UK (grant no. LOND1), which is funded by the UK MRC, Engineering and Physical Sciences Research Council, Economic and Social Research Council, Department of Health and Social Care (England), Chief Scientist Office of the Scottish Government Health and Social Care Directorates, Health and Social Care Research and Development Division (Welsh Government), Public Health Agency (Northern Ireland), British Heart Foundation and Wellcome Trust.

\section{Data Availability Statement}

The pre-print for this manuscript has been archived in the MedRxiv server: https://www.medrxiv.org/content/10.1101/ 2021.05.04.21256507v1.

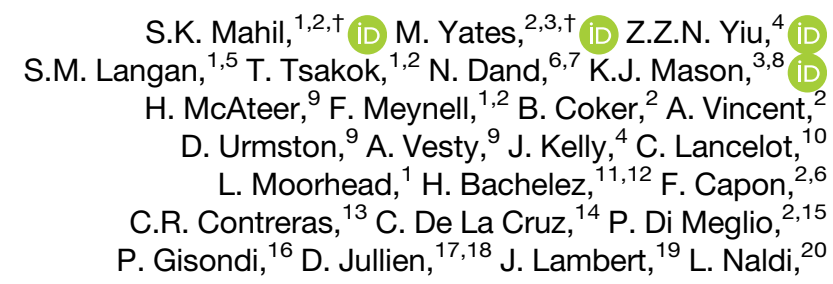

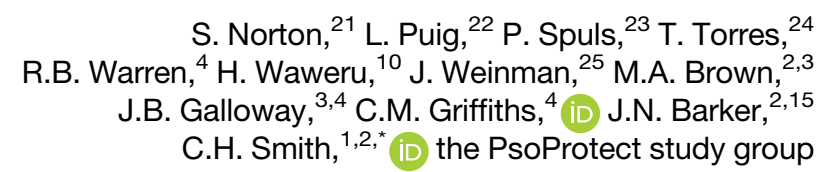
'St John's Institute of Dermatology, Guy's and St Thomas' NHS Foundation Trust and King's College London, London, UK, ${ }^{2} \mathrm{NIHR}$ Biomedical Research Centre at Guy's and St Thomas' NHS Foundation Trust and King's College London, London, UK, ${ }^{3}$ Centre for Rheumatic Diseases, King's College London, London, UK, ${ }^{4}$ Dermatology Centre,

Salford Royal NHS Foundation Trust, The University of Manchester,

Manchester Academic Health Science Centre, NIHR Manchester Biomedical Research Centre, Manchester, UK, ${ }^{5}$ Faculty of Epidemiology, and Population Health, London School of Hygiene and Tropical Medicine, London, UK, ${ }^{6}$ Department of Medical and Molecular Genetics, School of Basic and Medical Biosciences, Faculty of Life Sciences and Medicine, King's College London, London, UK, ${ }^{7}$ Health Data Research UK, London,

UK, ${ }^{8}$ School of Medicine, Keele University, Keele, UK, ${ }^{9}$ The Psoriasis Association, Northampton, UK, ${ }^{10}$ International Federation of Psoriasis Associations (IFPA), Bromma, Sweden, ${ }^{11}$ Department of Dermatology, AP-HP Hôpital Saint-Louis, Paris, France, ${ }^{12}$ INSERM U1163, Imagine Institute for Human Genetic Diseases, Université de Paris, Paris, France,

${ }^{13}$ Catedra de Dermatologia, Hospital de Clinicas, Facultad de Ciencias Medicas, Universidad Nacional de Asuncion, San Lorenzo, Paraguay,

${ }^{14}$ Clinica Dermacross, Santiago, Chile, ${ }^{15}$ St John's Institute of

Dermatology, School of Basic \& Medical Biosciences, Faculty of Life Sciences \& Medicine, King's College London, London, UK, ${ }^{16}$ Section of

Dermatology and Venereology, University of Verona, Verona, Italy,

${ }^{17}$ Department of Dermatology, Edouard Herriot Hospital, Hospices Civils de Lyon, University of Lyon, Lyon, France, ${ }^{18}$ Groupe de Recherche sur le Psoriasis (GrPso) de la Société Française de Dermatologie, Paris, France,

${ }^{19}$ Department of Dermatology, Ghent University, Ghent, Belgium, ${ }^{20}$ Centro Studi GISED, Bergamo, Italy, ${ }^{21}$ Psychology Department, Institute of Psychiatry, Psychology and Neuroscience, King's College London, London, UK, ${ }^{22}$ Department of Dermatology, Hospital de la Santa Creu i

Sant Pau, Universitat Autònoma de Barcelona, Barcelona, Spain,

${ }^{23}$ Department of Dermatology, Amsterdam Public Health/Infection and Immunology, Amsterdam University Medical Centers, Location AMC, Amsterdam, The Netherlands, ${ }^{24}$ Department of Dermatology, Centro Hospitalar do Porto, Porto, Portugal, ${ }^{25}$ School of Cancer and Pharmaceutical Sciences, King's College London, London, UK ${ }^{*}$ Correspondence: C.H. Smith. E-mail: catherine.smith@kcl.ac.uk †Joint first authors

\section{References}

1 Zylke JW, Bauchner H. Mortality and morbidity: the measure of a pandemic. JAMA 2020; 324: 458-459.

2 Pfefferbaum B, North CS. Mental health and the covid-19 pandemic. $N$ Engl J Med 2020; 383: 510-512.

3 Wright A, Salazar A, Mirica M, Volk LA, Schiff GD. The invisible epidemic: neglected chronic disease management during COVID-19. J Gen Intern Med 2020; 35: 2816-2817.

4 Mahil SK, Yates M, Langan SM et al. Risk mitigating behaviours in people with inflammatory skin and joint disease during the COVID-19 pandemic differ by treatment type: a cross-sectional patient survey. $\mathrm{Br} J \mathrm{Der}$ matol 2021; 185: 80-90. https://doi.org/10.1111/bjd.19755

5 Kroenke K, Spitzer RL, Williams JBW, Löwe B. An ultra-brief screening scale for anxiety and depression: the PHQ-4. Psychosomatics 2009; 50: 613-621. 
6 Mahil SK, Dand N, Mason KJ et al. Factors associated with adverse COVID-19 outcomes in patients with psoriasis-insights from a global registry-based study. J Allergy Clin Immunol 2021; 147: 60-71.

7 Gelfand JM, Armstrong AW, Bell S et al. National Psoriasis Foundation COVID-19 task force guidance for management of psoriatic disease during the pandemic: version 1. J Am Acad Dermatol 2020; 83: 1704-1716.

8 Pierce M, Hope H, Ford T et al. Mental health before and during the COVID-19 pandemic: a longitudinal probability sample survey of the UK population. Lancet Psychiat 2020; 7: 883-892.

9 Lamb RC, Matcham F, Turner MA et al. Screening for anxiety and depression in people with psoriasis: a cross-sectional study in a tertiary referral setting. Br J Dermatol 2017; 176: 1028-1034.

10 Read S, Morgan J, Gillespie D et al. Chronic conditions and behavioural change approaches to medication adherence: rethinking clinical guidance and recommendations. Patient Prefer Adherence 2020; 14: 581-586.

DOI: $10.1111 / j d v .17450$

\section{The significance of exanthems in COVID-19 patients hospitalized at a tertiary care centre}

Dear Editor,

Cutaneous manifestations have been associated with COVID-19 infection, and their significance in hospitalized patients remains unclear. $^{1-3}$ This study catalogues the exanthems observed in hospitalized COVID-19 patients to determine prevalence and inform clinicians in devising diagnostic and management strategies.

A retrospective review of 1216 adults hospitalized with laboratory-confirmed SARS-CoV-2 infection from 12 March 2020 to 31 May 2020 at a single institution was conducted. Keyword search of patient records combined with manual chart review by at least two dermatologists, with a third dermatologist for adjudication, identified patients with cutaneous manifestations based on chart documentation (Fig. 1).

Exanthems occurred in 39 patients $(3.2 \%)$ and were categorized clinically as morbilliform eruptions $(n=37 / 39$; $94.9 \%)$ and urticarial eruptions $(n=2 / 39 ; 5.1 \%)$ based on chart documentation and visual morphology (Table 1). Reviewers were aided by photographs for 26 patients (66.7\%) and inpatient dermatology consultations for 16 patients $(41.0 \%)$. A minority of patients $(n=7 / 39 ; 17.9 \%)$ had exanthems occurring within 14 days of COVID-19 symptom onset and thereby could be considered a possible viral reaction. The remaining 32 patients developed a rash more than 14 days after initial COVID-19 symptom onset. 21 patients $(n=21 / 32 ; 65.6 \%)$ developed a rash within 14 days of a clear causative medication exposure, suggesting a drug reaction aetiology. Dermatology reviewers identified likely culprit drugs in these patients, most commonly beta- lactam antibiotics $(n=17 / 21 ; 81.0 \%)$. Within the remaining 11 patients $(n=11 / 32 ; 28.2 \%)$, five patients were favoured to have a drug reaction within 28 days of culprit drug exposure based on clear documentation from chart review. The remaining six patients had insufficient data to adequately confirm the aetiology of their late-onset rash, though drug exposure was suspected based on the timeline.

Exanthems can present a diagnostic conundrum in distinguishing medication hypersensitivity versus viral infection as likely aetiologies. Exanthems due to viral infections typically present within 14 days of viral symptom onset. ${ }^{4}$ Skin rashes in COVID-19 are believed to present around the same time as other symptoms, typically during the first few days of fever and respiratory symptoms. ${ }^{1}$ In this cohort, only a minority of exanthems $(n=7 / 39 ; 17.9 \%)$ developed within 14 days of COVID-19 symptom onset to be considered for a viral rash. One of these seven patients was evaluated by dermatology and confirmed to have a drug hypersensitivity reaction by skin biopsy. The remaining six patients, $0.49 \%$ of all patients overall, could be potentially compatible with a viral aetiology for their exanthem based on timing of symptom onset.

Drug eruptions can result within 14 days of initial administration of the culprit drug but may also be delayed further by up to several weeks. ${ }^{5}$ The majority $(n=21 / 39 ; 53.8 \%)$ of exanthems in this cohort developed within 14 days of clear exposure to a common culprit drug and also after the 14-day window of COVID-19 symptom onset and resolved with cessation of drug use, supporting a medication-induced aetiology. In general, cutaneous drug reactions are estimated to develop in approximately $2 \%$ of patients, ${ }^{6}$ increasing to $8 \%$ with exposure to certain antibiotic groups. ${ }^{5}$ This is similar to the incidence of $3.2 \%$ ( $n=39 / 1216)$ found within this study.

In this study population of 1216 patients admitted with COVID-19, rashes from SARS-CoV-2 were exceedingly uncommon. These findings suggest that rashes previously ascribed to SARS-CoV-2 may have alternate explanations and highlight the importance of routinely considering medication exposures in the evaluation of generalized rashes occurring in hospitalized patients. Clinicians encountering exanthems in hospitalized patients with COVID-19 should maintain their usual standard of care for drug rashes ${ }^{7}$ in providing symptomatic treatment if necessary and considering withdrawal of the offending agent if a prolonged course of exposure is anticipated.

\section{Conflict of interest}

The authors have no conflicts of interest to declare.

\section{Funding source}

None. 